\title{
ECT joins the rotary club
}

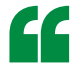

the divergent architectures

and protein compositions

are indicative

of ancient

adaptations

to different

environments

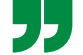

Electron cryotomography (ECT) can reveal the structures of macromolecular machines in bacterial cell envelopes. Using this transformative technology, Beeby et al. and Chang et al. now report structures for flagellar motors and the type IVa pilus machine (T4PM), respectively, to reveal biophysical principles that underlie bacterial motility.

Embedded in the cell envelope, flagellar motors harness a protonmotive or sodium-motive force to rotate the flagellum, which is a helical filament that is composed of flagellin subunits. Each flagellar motor contains a rotor and a stator; the heterooligomers of the stator
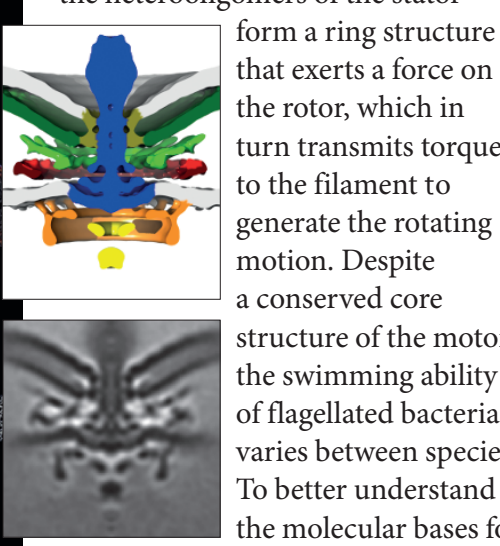
that exerts a force on the rotor, which in turn transmits torque to the filament to generate the rotating motion. Despite a conserved core structure of the motor, the swimming ability of flagellated bacteria varies between species. To better understand the molecular bases for these differences, Beeby et al. studied wild-type bacterial cells from three different species (Salmonella enterica, Vibrio fischeri and Campylobacter jejuni), together with genetic mutants that lack individual components of the flagellar motor. The torque exerted by the $S$. enterica motor has been estimated to be in the range of 1,300-2,000 pN nm, whereas the Vibrio spp. motor, which has more ready access to power owing to the sodium gradient in its marine environment, exerts an estimated torque of 2,000-4,000 pN nm. As C. jejuni has adapted to the high-viscosity environment of the epithelial mucus, its motor is expected to exert an even higher torque than that of $V$. fischeri. The ECT images showed that the stoichiometry of stator complexes in the stator ring differs between species, with 11 in S. enterica subsp. enterica serovar Typhimurium, 13 in V. fischeri and 17 in C. jejuni. Furthermore, the 'C-ring' (the cytoplasmic ring of the rotor) was wider in C. jejuni, with a radius of $26 \mathrm{~nm}$, than in $S$. Typhimurium and $V$. fischeri (with radii of $22 \mathrm{~nm}$ and $23 \mathrm{~nm}$, respectively).

The authors had previously shown that some flagellar motors have 'disc' complexes, of uncharacterized function, which are not found in enteric bacteria such as $S$. Typhimurium. The ECT images showed that $V$. fischeri and C. jejuni have a basal disc that is associated with the periplasmic face of the outer membrane; notably, the disc was wider in C. jejuni (with a radius of $42 \mathrm{~nm}$ ) than in $V$. fischeri (with a radius of $28 \mathrm{~nm}$ ). In the $V$. fischeri motor, the previously described $\mathrm{T}$-ring and $\mathrm{H}$-ring were present in addition to the basal disc. The C. jejuni disc complex also had additional components, a disc associated with the inner membrane and a periplasmic disc, but these were composed of different proteins to the T-ring and H-ring in V. fischeri, and also differed in complex architecture.

The authors suggest that disc complexes may function as scaffolds that enable motors to incorporate a larger number of stator complexes and, to increase leverage, at a wider radius, thereby applying more torque to the flagellum. Although the conserved basal disc suggests that a disc complex was present in an ancestral flagellar motor, the divergent architectures and protein compositions are indicative of ancient adaptations to different environments.

Whereas flagellar motors enable swimming by transmitting torque to the flagellum, the T4PM confers gliding motility to Myxococcus xanthus by powering the assembly and disassembly of the PilA subunits that form the helical pilus fibre, which results in a mechanism of extension and retraction. Chang et al. determined the architecture of the T4PM and mapped each component: an outer membrane pore (PilQ); a periplasmic ring associated with the outer membrane (PilQ and TsaP); a mid-periplasmic ring (PilQ and PilP); a lower periplasmic ring (PilN and PilO) and a cytoplasmic ring (PilM) that are connected by transmembrane coiled-coils (PilN and PilO); a cytoplasmic dome that is anchored in the inner membrane (PilC); and a cytoplasmic disc (PilB for pilus extension and PilT for pilus retraction). Interestingly, the structural data suggested that the T4PM extends and retracts the pilus using a rotating — rather than a fixed structure, with the rotation of PilC driving assembly and disassembly of PilA subunits.

Together, the studies demonstrate the power of combining ECT with bacterial genetics to elucidate the mechanisms of macromolecular machines.

Naomi Attar

ORIGINAL ARTICLES Beeby, M. et al. Diverse high-torque bacterial flagellar motors assemble wider stator rings using a conserved protein scaffold. Proc. Natl Acad. Sci. USA http://dx.doi. org/10.1073/pnas.1518952113 (2016) |Chang, Y. W. et al. Architecture of the type IVa pilus machine. Science 351, aad2001 (2016)

FURTHER READING Oikonomou, C. M, \& Jensen, G. J. A new view into prokaryotic cell biology from electron cryotomography. Nat. Rev. Microbiol. 14, 205-220 (2016) 